\title{
Descriptive Review of Energy Performance Evaluation Approaches
}

\author{
Siva Jaganathan ${ }^{\mathrm{a}}$, Abdul Hakim Mohammed ${ }^{\mathrm{a}}$, Mohd Shahril Abdul Rahman ${ }^{\mathbf{b}}$ \\ ${ }^{a}$ Centre for Real Estate Studies, Faculty of Geoinformation and Real Estate, Universiti Teknologi Malaysia, 81310 UTM Johor Bahru, Johor, Malaysia \\ ${ }^{b}$ Faculty of Geoinformation and Real Estate, Universiti Teknologi Malaysia, 81310 Johor Bahru, Johor, Malaysia \\ *Corresponding author: abdhakim@utm.my
}

\begin{abstract}
This study address energy performance evaluation uncertainities in design. To achieve energy efficiency in building, designer should incorporate energy performance evaluation approach to foresight energy performance failure during design. The research has evaluated and compares the capabilities of energy performances evaluation approaches namely computational fluid dynamic approach, optimization algorithm, and coupled approach. Furthermore, descriptive review unveils the practical obstacles and challenges designers encounter during design life cycle and proposes future direction to mitigate inundated energy performance gap.
\end{abstract}

Keywords: Energy, design, performance, building envelope, simulation, optimization

(C) 2016 Penerbit UTM Press. All rights reserved

\subsection{INTRODUCTION}

Designers has to cope with the trending needs and developments of energy management requirements to practice energy efficiency in post occupancy service life operation and maintenance. Simulation and optimization is an emerging trend and has become an integral part of design life cycle to address complex architectural, engineering and construction problems. To achieve energy efficiency in buildings, reduce post occupancy energy performance uncertainties and predict performance gap, designers urged to use integrated simulation and optimization approach in their design process. It is sporadic, use of simulation in energy performance evaluation and decisions to predict post occupancy energy performance in design decisions [1 \& 2].

Global survey about designer's understanding for energy performance evaluation during design posed various reasons as well as opposing opinions in the use of various simulation tools by industry practitioners.Most of the designers unaware and $52 \%$ of the designers disagreed to use complex simulation (i.e. CFD) in early design phase, because extensive boundary condition data input (temperature, weather data, geometrical inputs and thermo physical variables) [3]; lack of inter-operandi between application and with design tools that they have been using for construction documentation [4]; time consuming and expensive when developing alternative designs, and lastly it delays coping with timely design delivery [ $5 \& 6]$. Furthermore it's warrented, the cases that has been simulated for energy performance evaluation, more often the input data's were partially or fully based on assumtion rather using feedback/life cycle energy data [7]. Moreover, the historical data/case based data were not in ready-use form. This resulted huge variations in actual building energy consumptions [8]. Therefore, most of the tools required expert knowledge in operation and handling the complex interface made the process difficult (i.e. IES, TRANSYS, ENERGY PLUS, ECO-TECH, CFD-Fluent, eQuest, Green building studio, Energy_10). In contrast, studies also indicated the several applications lacks in compatibility and very few tools practically viable to meet sustainable building performance requirements $[13 \& 14]$. However, commercially popular and viable applications enable the designer to predict only, indoor airflow turbulence, HVAC dimension (plant), thermal comfort, day lighting levels and impact on acoustic [15 \& 16]. But they are lack in quantify the design influenced post occupancy energy performance gap. Despite all these drawbacks, it is inevitable the expert systems like simulation knowledge in design has paramount importnace in order to reduce design influenced performance failures. Integerating simulation in early phase of design pave the way for designer to foresight energy consumption and improve energy performance efficiency.

This paper set the focus to answer the research question that what are all the measures that has been taken to improve energy performance based design approach to abridge performance gap between design and actual building?. This paper presents the research works related to energy performance evaluation, performance uncertainities and prediction of energy consumption approaches. These methods include evaluation by computational fluid dynamic approach; using optimization algorithem and couple energy performance evaluation approach. To even further enrich for reader view, this paper also provides comparison of strength and weakness these approaches for design inclusion. Content of this paper organized as follows. Section 2, presents recent research work energy performance evaluation. Section 3 presents the discussion and future research points. Section 3 presents the conclusion.

\subsection{METHODS THAT INVESTIGATED ENERGY PERFORMANCE UNCERTAINITIES}

Over the past fifty years, hundreds of simulations based optimization applications have been developed to address performance evaluation and to rationalize design decisions (i.e. Green Building, Studio, BSim 4.4, DeST 2.2 (Designer simulation tool kit), ECOTECH, Enter-Win 
(Developed by Texas university), HAP-4.2a (Hourly Analysis Program), HEED, DOE-2, eQuest, Energy, Energy Plus, BLAST, ESP-r, IES, Power Domus-1.5, SUNREL 1.14, Tas, TRACE-700, TRANSYS etc.). The energy performance evaluation means, applying scientific criteria, methods and procedures to make correct evaluation on energy performance. The reviews based on the viable application potential by designers, limitations, performance prediction and feasible to incorporate. Most of the suggested simulation methods use physical principle to compute thermal dynamics, and energy behavior of whole building. They are mostly derived from operation research (OR), artificial intelligence (AI) and many other approaches (i.e. stochastic optimization, fuzzy reasoning, swarm intelligence, multi criteria optimization, meta-heuristics, exact optimization methods and experimental design). These approaches are highly comprehensive and complex in nature for the designer to use. Designer entrenched background towards design process and material application, demonstrated their in ability to use them in design for performace evaluation. Several measures that has been initiated by agencies who certify building subscribed to energy efficiency. One of them was to give high bonus points for designer, if their design decision were made using simulation and optimization to enhance post occupancy energy performance $[9,10,11 \& 12]$.

Hundred of application were been developed for evaluating energy performance, renewable energy and sustainability in buildings such as DOE, Energy plus and ESP.r. Some of them widely used by designers and used to develop energy efficiency standards. Since these tool are based on physical principal, to achive acuurate simulation out come, they require details of building environmental parameters as the prime input data. As mentioned, these input data's were not ready to use form. On one hand its not available for many organization and difficult to obtain for large buildings. This warrentd low accuracy in simulation lead to post occupancy performance uncertinities. De Wit [17] investigated domain expertise of physical properties, responsible for those sustainable inputs in design team at large. According to De Wit, in current design practice performance evaluation not explicity quantified. S. Kim and Augenbroe [19] defined several areas of uncertainties and quantified the performance gap using probabilistic approach.

Hopfe et al. [21] compared the energy consumption results of four-simulation approaches using uncertainty analysis. Comparison of the results led to the development of an influential design parameters energy model for cooling and heating. S. De Wit and Augenbroe [18] addressed the effect of variations in heat transfer variables on thermal comfort and energy consumption that rationalize design decisions. Hopfe et al. [20] showed the effect of variations in physical parameters in an energy model on heating and cooling energy use in relation to unmet building loads. Heiselberg et al. [22] designed sensitivity technique to optimize building energy consumption to meet green building standards. Dominguez-Munoz, Cejudo-Lopez, and Carrillo-Andres [23] proposed methodology to model uncertainties in building energy consumption and greenhouse gas emission under climate change. Rysanek and Choudhary [24] studies identified a limited number of energy-saving measures using uncertainty techniques to reach cost effectiveness to reduce energy consumption.

Wang and $\mathrm{Xu}$ [25] simplified physical characteristics of buildings such as building envelope to implement the predictions. Wang, Mathew and Pang [26] explored significance of climate, physical and mechanical system parameters influence on energy consumption. Tian and De Wilde [27] studied peak cooling load calculation using Monte Carlo analysis. Reviewed literature suggest that each one of these method unique in its performance evaluation. They are mostly lacks in holistic approach, that can quanitify total building performance. On one hand, these approach no where come closer to practical application.

\section{Energy Performance Evaluation Using Simulations}

More than 20 major energy simulation programs were used in design. Computer simulation allow designer to test their design concepths before being incorporated into building. In this context building performance analytical model can be applied to solve complex problems and to reduce energy cost. Building simulation expands the concept of performance simulation with the help of building performance simulation, the designer can define the parameter that have an influence on overall performance. There are many simulation programs that could be used to conduct performance evaluation such as TRNSYS IDA_ICE, DOE-2, Energy-Plus, APACHE, Ecotect, Radiance and CFD based applications. Among CFD based programs are the one mostly used.. As stated by Wetter and Wright [29] CFD based complex simulation programs generally involve coding features. It is a promising multi-physic, three-dimensional approach, and highly recommended for microscopic prediction. CFD models have number of clear advantages as compared with other approaches as opposed to most experimental techniques including Particle Image Velocimetry (PIV). It has full potential to control dynamic boundary conditions. In addition, CFD is most appropriate for stratified simulation such as indoor air quality, thermal transient flow and natural ventilation. Transient models work with any one of these principles, which are steady state RANS method, K-epsilon and laminar turbulent. As mentioned CFD approach highly relies on detailed 3D description, fine meshing qualities (smaller the mesh longer the computing time). Therefore, it is advisable to apply CFD technique only on specific constituents in building components to understand the energy performances.

\section{Energy Performance Evaluation By Optimzation}

Computational optimization is applied in various field of engineering for solving complex construction and engineering problems. The use of computational optimization and performance modeling are left to designer prerogative and their experience in order to achieve best solutions [30]. Because, application optimization in design requires engineering knowledge, and the designer have to work collaboratively in order to solve building performance optimization. Simplifying the optimization process itself is the niche research that could facilitate designers to develop various possibilities to achieve energy performance efficiency.

Research studies extensively investigated the use of evolutionary meta-heuristic multi-objective optimization algorithm (i.e. genetic algorithm, artificial neural network, ant colony optimization, Pareto optimization and Particle swarm optimization) applications for designing energy efficiency building, net-zero energy buildings and low carbon emission design. Holst [31] used genetic optimization approach and energy plus in their case study to reduce energy use and developed comfort for metric percentage. The variables used to investigate included WWR, thermal properties, insulation and shading devices. Coley \& Schukat [32] used genetic algorithm combined with human judgment architectural appeal to assess post energy use through visual manners thereby enabling the user to choose based on performance. J.H. Wright et al. [33] used GA for simultaneous optimization of the fabric construction, HVAC system size and the control strategy. G. Augenbore [34] used GA to minimize annual energy use. Similarly Bambrook et al., [35] used brute-force method and e-quest to minimize energy use by means of varying fabric properties such as building shape, glazing properties and kind of ventilation method 
used. R. Evins [36] examined the performance role of double skin facade in facade design and explored trade-off between cooling load and heating load by using multi objective genetic algorithm. Torres \& Sakamoto [37] used genetic algorithm to optimize day light by wide means of parameters that cover window size, placement, shading and reflectance to reduce use of energy using non-linear real time lumped parameters to address transient temperature flow. Most of the examined studies used genetic algorithm to assess performance by various physical functions of buildings, however, there is ayto of evidence found, that its applicable in $\mathrm{r}$.

\section{Energy Performance Evaluation By Coupled Approach}

Integration of programs with different purposes in energy simulation eliminates many model assumptions as in separate applications and make it possible to avoid errors and improve the quality of simulation if coupled with meta-heuristic algorithm, which has been noted to produce better results of performance predictions [38]. The coupled approach evolved by Negarao [39] is considered mostly straightforward, reliable and efficient in evaluating energy performances. However, coupling approach is more complex and it can be applied when complex conditions arise. For example, the need for coupling method determined based not only on building environmental characteristics. It can be determined by various engineering and design constraints such as sensitivity of indoor air movement and air temperature stratification to thermal boundary conditions and building energy requirements. According to Y. Fan, K. Ito [40] one of the prevalent methods to have emerged in coupling between two programs is mostly made possible by programmatic approach such as running script file for CFD and BES components.

Based on the stated constraints, Z. Zhiqiang et al. [41] considered exchange protocols to improve the cooling/heating loads and comfort predictions. Similarly, Zhai and Chen [42] verified convergence of a solution obtained by a coupling approach based solely on thermal aspects. Testing several exchanges parameters such as convective heat transfer coefficients or heat fluxes from CFD to BES is claimed to be most stable approach. Q. Chen [43], tested script files of surface temperature from building energy simulation to computational fluid dynamic in thermal transient flow and energy use dynamics. Coupling approach predicts over cooling accurately. However, coupling approach was not prevalent amongst design practitioners. In addition, designers do not have efficient tools and adequate skills to pursue their design life cycle. Learning skills is an ability of the learner to learn new process, access, manage, organize and ask questions in order to have productive study skills by using appropriate learning tools and strategies. This could also be related to the ability to learn independently and develop attitude to learn. It is also related to the skills on where and how to get information from available sources and also be able to manage and organize the information effectively [12]

\subsection{DISCUSSION}

This paper presents the energy performance evaluation design approach using simulation, optimization and coupled approach. As the investigation of past ten years of research works clearly indicated, there is persistent interest among designers and construction practitioners to apply these methods in their design life cycle. The cited research works investigated various energy related performances and tested many physical variables in combination (i.e. glazing and orientation). Holistic approach by various engineering methods, CFD, optimization and coupled has shown to address performance issues in harmony. The major obstacle to solve issues is that a whole building simulation and optimization program is computationally expensive, thus making selection of an efficient algorithm quite critical. Environmental impact, energy consumption and many other performance issues were targeted in the optimization study. Building envelop parameters, orientation, climate data, geometry, and glazing options are the most researched variables pertaining to performance evaluation. In a review of opinions, most of the simulation work at least bothered to consider post occupancy energy data for case based comparison. Further to this, facilities management inputs were minimal in performance evaluation during design phase. Lastly, lack of appropriate integrated tools has also led several research works to develop practically unreliable methods by designers. Most of the studies signled out variable's in their investigation for developing design integrated simulation and optimaztion. For instance, there is no study conducted using post occuapancy energy performance data as base line to perform energy performance evaluation in design. This disable the designers to work in tandem with the line of post occupancy performance requirements and optimization.Despite this issues, it is also showing, there is an increasing concern that use of computation simulation during the design is vital means to achieve economically viable solution for energy performance optimization. Sustainable and green building ratings driven designers to inherit the such a complex knowledge of performance evaluation in design approach. This review concludes that it is time for researchers to simplify the performance evaluation approach in design as well as develop an robust, prediction model (Figure 1) that incorporates post occupancy energy data insights within the realm of the design process. 


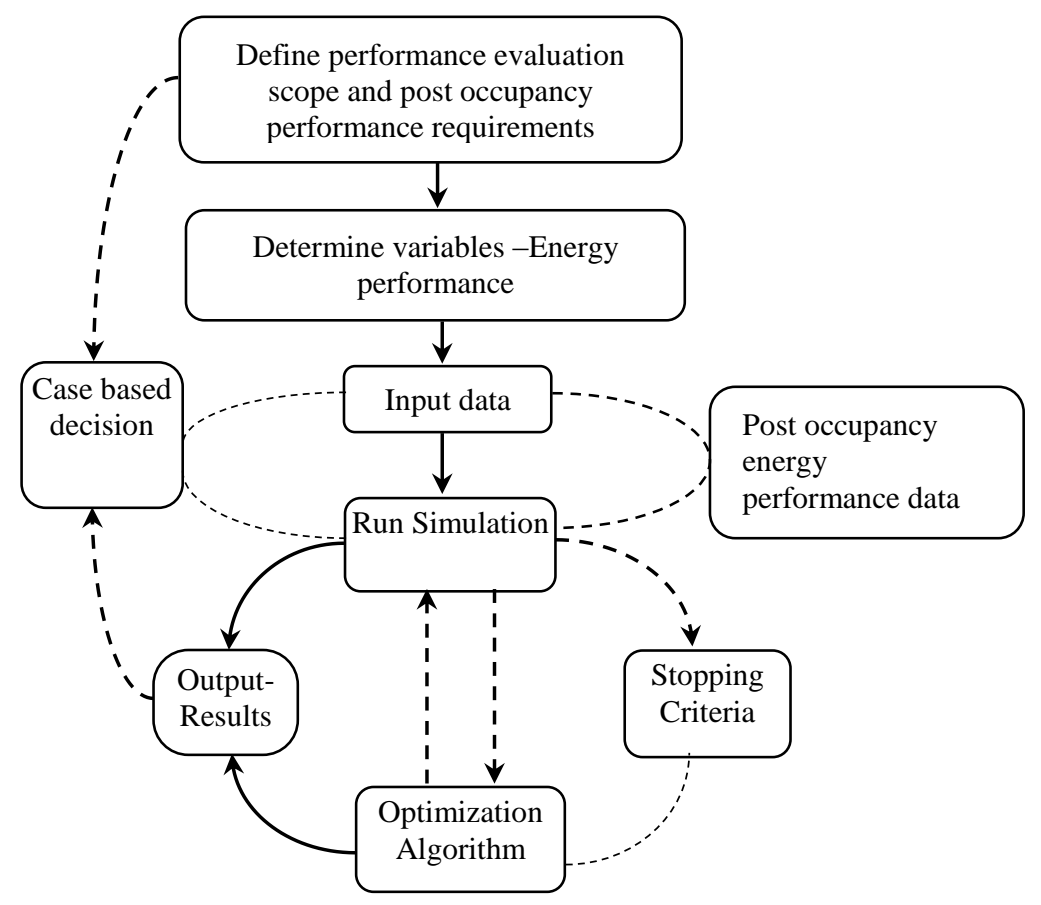

Figure 1 Schematic flow diagram for propoed energy performance evaluation

\subsection{CONCLUSION}

With the growing trend in incorporating sustainable evelopment, the design design of performance based evaluation will surely gain attentions. Building performance based simulation, optimization and coupled approach not only reveal the interaction between building and HVAC systems and out door climate, but also make possible to integrated post occupancy energy perofmance data to improve design decisions. We have shown how important the performance evaluation using these different approaches and lead to completely lead new building desiegn philosophy and design methodology. The overview presented in this paper aims introduce possibilities of existing simulation, optimization and coupled approaches to improve performance gap. This study proposed an integrated approach that facilitated both designer's and feasible to incorporated post occupancy energy performance data for better design decision and mitigate performance gap.

\section{Acknowledgement}

Author gratefully acknowledges researchers from Centre for Real Estate Studies (CRES), UTM, for their significant comments and criticisms in completing this article.

\section{References}

Adeleke, M. H. (2006). An Appraisal of Curriculum Implementation in Nigeria, Lagos: Macus Publication.

Adeyemi, T. O. (2008). The Availability of Teaching Manpower in Technical Colleges in Ondo and Ekiti States, Nigeria: A Comparative Analysis. MiddleEast Journal of Scientific Research, 3(4), 179-189.

Aina, O. (2009). Three Decades of Technical and Vocational Education and Training in Nigeria. Ile-Ife: Obafemi University Press Ltd.

Akuezuilo, E. O. (2007). The New 9-year Basic Science and Technology Curriculum and Challenges of its Implementation. Journal of Curriculum and Instruction, 6(2): $2-5$.

Arbuckle, J. L. (2007). AMOS 16.0 User's Guide. Spring House. PA: Amos Development Corporation.

Augenbroe, G. (1992). Integrated Building Performance Evaluation In The Early Design Stages. Building and Environment. 27 (2): $149-161$

Bambrook. S. M., A. B. Sproul, D. Jacob. (2011). Design Optimization For A Low Energy Home in Sydney. Energy and Building. 43 (7): 1702 -11.

Brans, J.P., B. Mareschal. (1995). The PROMETHEE VI procedure. How to Differentiate Soft From Hard Multicriteria Problems In The Discrete Case. Journal of Decision Systems. 4 (3): 213-224.

Bouchlaghem, N., (2000). Optimising The Design Of Building Envelopes For Thermal Performance. Automation and Construction. 10: $101-112$.

Carmody, J, S. Selkowitz, E. Lee, D. Arasteh, and T. Willmert. 2004. Window Systems for High-Performance Buildings, Norton \& Company, New York.

Education for Emergent Globalization, Relevance and Sustainable Economic Development. International Journal of Vocational and Technical Education, 2(4), 55-61

Castro Lacouture. D., J. A. Sefair, L. Flórez, A. L. Medaglia. (2009). Optimization Model For The Selection Of Materials Using A LEED-Based Green Building Rating System in Colombia. Build Environ. Building and Environment. [6]: 1162 -70.

Coley, D.A., S. Schukat. (2002). Low-Energy Design: Combining Computer-Based Optimisation And Human Judgment. Building and Environment. 37: 1241-127.

De Wit, S., and G. Augenbroe. (2002). Analysis of Uncertainty in Building Design Evaluations and Its Implications. Energy and Buildings. 34: 951-958

Domínguez-Muñoz, F., J. M. Cejudo-López, and A. Carrillo- Andrés. 2010. Uncertainty in Peak Cooling Load Calculations. Energy and Buildings. 42 (7): 
$1010-1018$

Donn. M. 2004. Simulation Of Imagined Realities Environmental Design Decision Support Tools In Architecture. Ph.D. Thesis. School of Architecture, Victoria University, Wellington.

Fesanghary. M., S. Asadi, Z.W. Geem. (2012). Design of Low-Emission And Energy Efficient Residential Buildings Using A Multi-Objective Optimization Algorithm. Building and Environment. 49: 245-50

Foucquier. A., S. Robert, F. Suard, L. Stéphan, A. Jay. (2013). State Of The Art In Building Modelling And Energy Performances Prediction: A Review. Renew Sustainable Energy. 23: 272-88.

Gao, S., Mokhtarian, P. L., and Johnson, R. A. (2008). Non-Normality of Data in Structural Equation Models. Transportation Research Board's $87^{\text {th }}$ Annual Meeting, January. Washington D.C

Grob, R.F., M. Madjidi. (1997). Commissioning and fault detection of HVAC systems by using simulation models based on characteristic curves, in: clima-2000 conference, Brussels.

Heiselberg, P., H. Brohus, A. Hesselholt, H. Rasmussen, E. Seinre, and S. Thomas. (2009). Application of Sensitivity Analysis in Design of Sustainable Buildings. Renewable Energy. 34 (9): 2030-2036

Holst J. N. 2003. Using Whole Building Simulation Models And Optimizing Procedures To Optimize Building Envelope Design With Respect To Energy Consumption And Indoor Environment. Eighth International IBPSA Conference, Eindhoven, Netherlands. In: Proceedings of the Building Simulation.

Hopfe, C., and J. Hensen.(2011). Uncertainty Analysis in Building Performance Simulation for Design Support.” Energy and Buildings. 43 [10]: 2798 -2805.

Hopfe, C., C. Struck, P. Kotek, J. van Schijndel, J. Hensen, and W. Plokker. (2007). Uncertainty Analysis for Building Performance Simulation - A Comparison of Four Tools. Proceedings of the 10th IBPSA Building Simulation Conference. Beijing, China. 1383-1388.

Iassinovski, S., A. Artiba, V. Bachelet, and F. Riane. 2003. Integration of Simulation And Optimization For Solving Complex Decision Making Problems. International of Journal Production and Economics. 85: 3-10.

Nguyen. A., S. Reiter, P. Rigo. (2014). A Review On Simulation-Based Optimization Methods Applied To Building Performance Analysis. Applied Energy. 113: $1043-58$.

Mahdavi, A., S. Feurer, A. Redlein, and G. Suter. (2003). An Inquiry Into The Building Performance Simulation Tools Usage By Architects In Austria, Proceedings of the Eighth IBPSA Conference. Eindhoven, Netherlands. 777-784.

Mahdavi, A., S. Feurer, A. Redlein, and G. Suter. (2003). An Inquiry Into The Building Performance Simulation Tools Usage By Architects In Austria, Proceedings of the Eighth IBPSA Conference. Eindhoven, Netherlands. 777-784.

Fisher D. E. , M.J. Witte, J. Glazer. (2001). Energy Plus: Creating A New-Generation Building Energy Simulation Program. Energy and Building. 33: 319-31.

Kim, S. H., and G. Augenbroe. 2013. Uncertainty in Developing Supervisory Demand-Side Controls in Buildings: A Framework and Guidance. Automation in Construction. 35: 28-43.

Rysanek, A., and R. Choudhary.(2013). Optimum Building Energy Retrofits Under Technical and Economic Uncertainty. Energy and Buildings. 57: 324-337.

Wright J, H. Loosemore, R. Farmani. 2002. Optimization of Building Thermal Design And Control By Multi-Criterion Genetic Algorithm. Energy and Buildings. 34 (9): 959-972

Hox, J. J and Bechger, T. M. (1998). An Introduction to Syructural Equation Modeling. Family Science Review, 11, 354-373.

ILO-International Labor Organization. (2008). Recognizing Ability: The Skills and Productivity of Persons with Disabilities. International Labour Office, Skills and Employability Department.-Geneva: ILO, 2008.

James, A., Fraces, L., Elaine, C., Wynn, C., Jim, H. and Jack, W. (2007). Models for Curricular Materials Development: Combining Applied Development Processes with Theory. Journal of Science Education and Technology, 16(6), 491.

Kennedy, O. O. (2011). Reappraising the Work Skill Requirements for Building Technology Education in Senior Secondary School for Optimum Performance in Nigeria. European Journal of Applied Sciences, 3(2), 46-52.

Kline, R. B. (1998). Principle and Practice of Structural Modeling. New York: Guilford Press.

Mailea, T., V. Bazjanac and M. Fischera. (2012). A Method To Compare Simulated And Measured Data To Assess Building Energy Performance. Building and Environment. 56: 241- 251

McLeish, A. (2002). Employability Skills for Australian Small and Medium Sized Enterprises. Report of the Interviews and Focus Groups with Small and Medium Enterprises.

National Policy of Education. (2004). Federal Republic of Nigeria. Lagos: NERDC Press.

Oduolowu, E. A. (2007). A Comparison of the Universal Basic Education (UBE) Programme in Nigeria and the Grundskola of Sweden. Essays in Education, 20, 9093.

Olayinka, O. and Oyenuga, O.A. (2010). Integration of Automobile Technological Developments into Nigeria Technical College Motor Mechanics Work Curriculum. Academic Leadership: The Online Journal, 8(2), 1-11.

Oloruntegbe, K. O., Agbayewa, J. O., Adodo, S. O., Adare D., and Laleye, A. M. 2010. Reconceptualization of African Vocational and Technological.

Partners for 21st Century Skills. (2009). P21 Framework Definitions.Available: http://www.p21.org/storage/documents/P21_Framework_Definitions.pdf.

Psacharopoulos, G and Woodhall, M. (1997). Education for Development: An Analysis of Investment Choice. New York Oxford University Press.

Sakamota, A. and Powers, P. A. (1995). Education and the Dual Labour Market for Japanaese Men in American Sociological Review, 60(2), P. $222-246$.

Schultz, T. W. (1971). Investment in Human Capital. New York. The Free Press.

Wan-Mohammed, W. A and Yunus, M. H. (2009). The Inculcation of Generic Skills among Juveniles through Technical and Vocational Education. Us-China Education Review, 6(4), 56-61.

Wang, W. 2005. A Simulation-Based Optimization System for Green Building Design. PhD Thesis. Concordia University.

Wang. L., N. H. Wong. (2008). Coupled Simulations For Naturally Ventilated Residential Buildings. Automation in Construction. 17:386-98.

Wang. W., H. Rivard, R. Zmeureanu. 2005. An Object-Oriented Framework For Simulation-Based Green Building Design Optimization With Genetic Algorithms. Advanced Engineering Information. 19(1): 5-23.

Wang. L., P. Mathew, X. Pang. (2012). Uncertainties In Energy Consumption Introduced By Building Operations And Weather For A Medium-Size Office Building. Energy and Building. 53: $152-8$.

W. Tian, W., P. de Wilde.(2011). Uncertainty and Sensitivity Analysis Of The Performance Of An Air-Conditioned Campus Building In The UK Under Probabilistic Climate Projections. Automation and Construction. 20: 1096-1109.

Wang Shengwei, Yan Chengchu, Xiao Fu. (2012). Quantitative Energy Performance Assessment Methods For Existing Buildings. Energy and Building. 55: $873-88$.

Wetter M, J. Wright. 2004. A Comparison Of Deterministic And Probabilistic Optimization Algorithms For Nonsmooth Simulation-Based Optimization. Building and Environment. 39: 989 - 99.

Wilson, K. (2008). Entrepreneurship and Higher Education. Entrepreneurship Education in Europe. European Foundation for Entrepreneurship Research. P1-9.

Zelenay, K., K. Perepelitza, and D. Lehrer, (2011). High-Performance Facades Design Strategies And Applications in North America and Northern Europe. California Energy Commission, Publication number: CEC-500-99-013 